\title{
Enlarged Parent Artery Lumen at Aneurysmal- Neck Segment in Wide-Necked Distal Internal Carotid Artery Aneurysms
}

\author{
Jong Won Lee², Jung Min Woo, B.S.', Ok Kyun Lim, B.S.', Ye-eun Jo, B.S.', Jae Kyun Kim, MD, PhD², \\ Eun Sang Kim, M.B.A. ${ }^{4}$, Deok Hee Lee, MD, PhD ${ }^{1,2}$
}

Purpose: Hypothesizing that the parent artery (PA) diameter of the aneurysm-neck segment is larger than those of normal segments, especially in wide-necked aneurysm cases, we conducted 3D angiographic analyses in wide-necked aneurysm cases focusing on the luminal morphologic change of the PA.

Materials and Methods: Under the approval of local IRB, we enrolled 26 patients with distal internal carotid artery (ICA) aneurysms, which were treated with stent assisted coiling. The PA diameters along the centerline were measured at 6 points with built-in software by two observers. Those 6 points were $\mathrm{P} 1$ and $\mathrm{P} 2$ proximally, $\mathrm{P} 3$ and $\mathrm{P} 4$ at the aneurysm ostium margins, and $\mathrm{P} 5$ and $\mathrm{P} 6$ distally. We performed an ANOVA test and a Bonferroni method for post hoc analyses. Linear regression analysis was performed to find any morphologic influencing factors.

Results: There were 20 distal ICA aneurysms out of 26 consecutive cases after exclusion. The differences in diameter at each point were statistically significant $(p<0.0001)$. On post hoc analyses, the difference between P4 and P5 was significant both in maximum and mean PA diameters $(p<0.0001$ and $p<0.001$, respectively). Multivariate analyses failed to reveal any morphological influencing factor.

Conclusion: PAs harboring a wide-necked aneurysm requiring stent assistance for coiling showed significant enlargement of the lumen, especially at the distal transition segment of the aneurysm ostium and the PA.

Key Words : Cerebral aneurysms; Parent artery; Cerebral angiography; Intracranial stenting; Vascular morphology

This research was supported by a grant (HO13C0006) from the Osong Innovation Center funded by the Ministry of Health \& Welfare, the Republic of Korea.

'Department of Radiology and Research Institute of Radiology, Asan Medical Center, Seoul, Korea

${ }^{2}$ Department of Medicine, University of Ulsan College of Medicine, Ulsan, Korea

${ }^{3}$ Department of Radiology, Chung-Ang University College of Medicine, Seoul, Korea

${ }^{4}$ Angiovention, Gyeonggi-do, Korea

Received March 19, 2015;

accepted after revision July 6, 2015.

Correspondence to: Deok Hee Lee, MD, PhD, Department of Radiology, Research Institute of Radiology, Asan Medical Center, University of Ulsan College of Medicine, 88, Olympic-ro 43-gil, Songpa-gu, Seoul 138-736, Korea.

Tel. 82.2.3010.5944 Fax. 82.2.476.0090 E-mail: dhlee@amc.seoul.kr

This is an Open Access article distributed under the terms of the Creative Commons Attribution Non-Commercial License (http://creativecommons.org/licenses/by-nc/3.0) which permits unrestricted non-commercial use, distribution, and reproduction in any medium, provided the original work is properly cited. 
Endovascular coiling has become popular for the treatment of both ruptured and unruptured cerebral aneurysms. To prevent the coil loops from protruding into the parent artery (PA) lumen, self-expanding stents are placed and serve as physical scaffolds for widenecked aneurysms [1]. However, malapposition of the stent mesh to the PA wall is not infrequent, especially with closed-cell-type stents when the PA has some tortuosity [2]. Open-cell-type stents are also not completely free from the malapposition issue because of underlying morphologic abnormality of the PA at the aneurysm neck segment.

In our clinical experience with wide-necked aneurysms, we could observe variable degree of PA lumen enlargement or dilatation at the aneurysm-neck segment compared with the proximal or distal segments of the PA. We thought that this kind of parent artery morphologic abnormality associated with the aneurysm exaggerated malapposition problem (Fig. 1). Nevertheless, few reports have focused on geometric changes of the PA itself in the evaluation of aneurysmal disease. Based on the hypothesis that the PA diameter of the aneurysm-
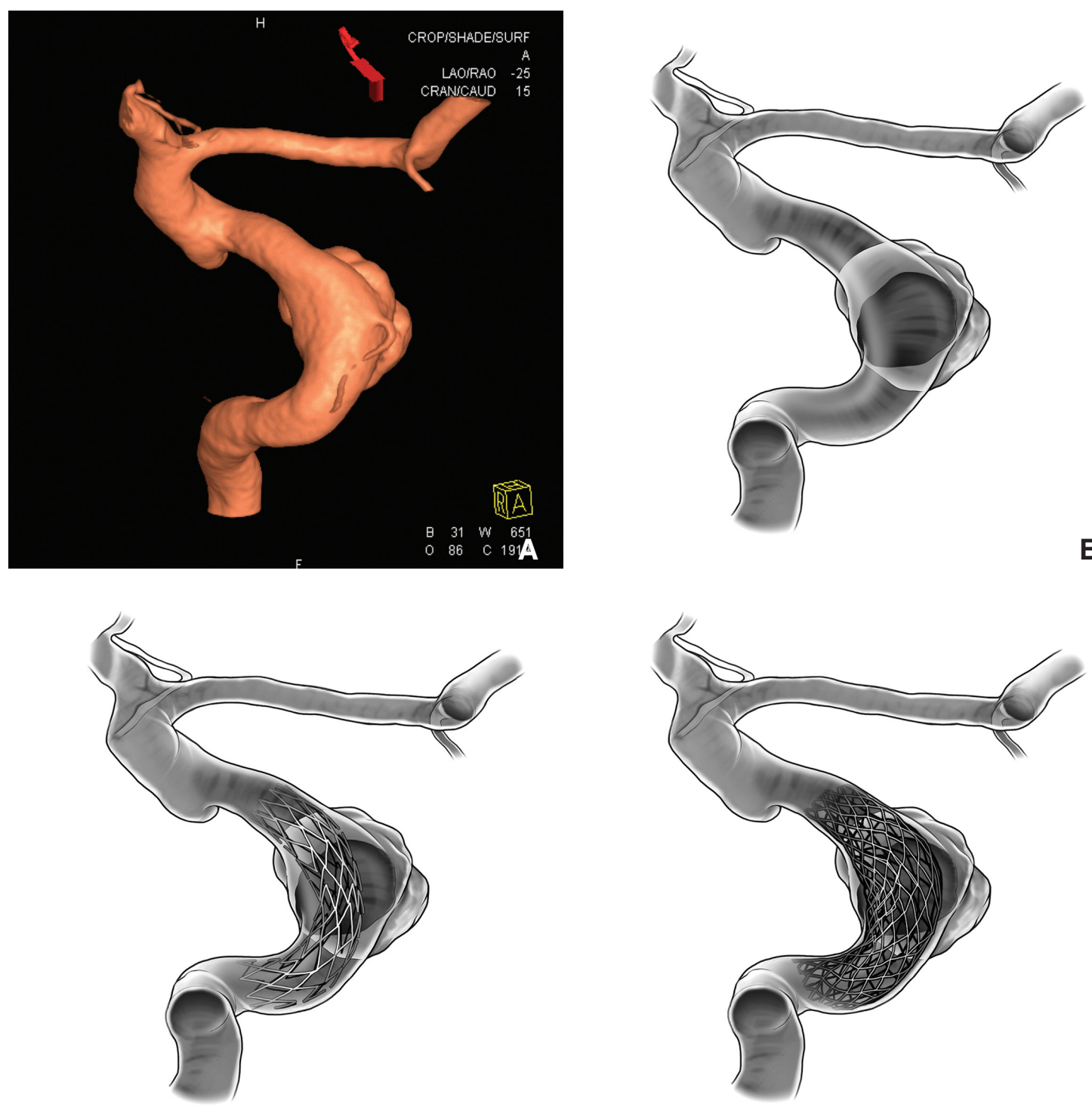

C

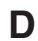

Fig. 1. Angiography was performed in a patient with an incidental aneurysm. (A) Volume rendering (VR) image of the 3D angiography showing a lobulated, wide-necked aneurysm at the ophthalmic segment of the right internal carotid artery. (B) A drawing in transparent view demonstrates the ostium of the wide-necked aneurysm and associated segmental distention of the parent artery lumen as well. (C) A self-expanding stent is placed across the neck of the aneurysm. We exaggerated the malapposition problem in this particular picture. We may improve the apposition by choosing a larger-diameter stent to fit into the enlarged segment. (D) However, due to the size limitation in the diameter and intrinsic tortuosity of the parent artery, there could be significant malapposition, even with significant oversizing of the stent. 
neck segment is wider than that of the proximal or distal PA segments, especially in cases of wide-necked aneurysms, we conducted a 3D angiographic data analysis to see whether there was a significant difference in the PA diameters at the aneurysm neck segment in cases of wide-necked distal internal carotid artery (ICA) aneurysms.

\section{MATERIALS AND METHODS}

\section{Patients and angiographic data}

This retrospective image analysis study was approved by our local institutional review board, and individual patients' informed consent was waived. To identify patients with wide-necked ICA aneurysms, we reviewed our neurointerventional database and extracted cases of distal ICA aneurysms treated with stent-assisted coiling from November 2011 to June 2013. We regarded an aneurysm neck that required stent assistance for elective coil embolization large enough for the fulfillment of the generally-accepted concept of wide-necked aneurysms.

The decision to use a self-expanding stent for the coiling of a wide-neck aneurysm was made according to the individual operator's discretion; however, a stent was generally considered in aneurysms with a neck size exceeding $4 \mathrm{~mm}$ in maximum dimension and/or with a dome-to-neck ratio smaller than 1.5. In some cases that met the criteria, stents were not used because other assisted techniques were feasible, and these cases were excluded from this study to simplify the case enrollment process. To eliminate or minimize possible compounding factors for accurate morphologic analyses, other exclusion criteria were applied, and were as follows: (a) the stent was placed to bail out inadvertent herniation of the coil loops during a nonstent assisted coiling procedure, (b) a branching vessel was involved by the aneurysm, (c) a major branching vessel was present within $7 \mathrm{~mm}$ from the proximal and distal margins of the aneurysm ostium, and/or (d) no available 3D angiographic data was available for vessel analysis, even in cases where stent-assisted coiling was performed.

\section{Angiographic data acquisition}

Angiographic examinations were performed using a biplane neuro-angiography unit (Siemens Artis Zee, Siemens Healthcare, Erlangen, Germany) with an image intensifier matrix of $2048 \times 2048$. Using a right femoral artery approach, conventional internal carotid angiograms were obtained after injection of $6-8 \mathrm{~mL}$ of iodinated contrast medium (Visipaque 270, GE Healthcare, Princeton, NJ) at a flow rate of 4-6 mL/sec.

For rotational angiography, after collimating the patient's head to isolate the aneurysmal region, the $\mathrm{C}$ arm was rotated over a 200-degree range at a rate of 40 degrees/sec for 5 seconds. Contrast medium was injected at a flow rate of $2-3 \mathrm{~mL} / \mathrm{sec}$ for $6 \mathrm{sec}$. Data obtained were then transferred to an external processing workstation (Syngo Workplace, Siemens Medical Solutions) to generate a volume rendering (VR) image using the vendor supplied 3D software (Inspace, Siemens Medical Solutions).

\section{Morphologic definition and analysis methods}

Before measurement, we defined several morphologic parameters for the aneurysm itself and its PA segment (Fig. 2). The interface between the aneurysm sac and the PA was the ostium (the term, "neck", was reserved for external morphology of the aneurysm, which was defined during surgery) [3]. 'Maximum aneurysm sac diameter' denoted the longest distance $(\mathrm{mm})$ of the sac regardless of the dimension. 'Maximum and minimum ostial distances' denoted the length along the longest and shortest dimensions, respectively, of the aneurysm ostium on cross section. 'Ostial area' denoted the cut-surface area $\left(\mathrm{mm}^{2}\right)$ of the ostium. The aspect ratio was calculated by dividing the aneurysm height by the maximum ostial distance. The dome-to-neck ratio was calculated by dividing the dome width by the maximum ostial distance.

By drawing two separate points along the PA, one proximal to the aneurysm and the other distal to the aneurysm, a centerline was automatically obtained on the workstation (Fig. 2A). The detailed measurement method is described below. The PA segment of interest was arbitrarily designated from a point $7 \mathrm{~mm}$ proximal to the proximal margin of the ostium to $7 \mathrm{~mm}$ distal to the distal margin of the ostium along the centerline (Fig. 3B). That arbitrary length of $7 \mathrm{~mm}$ was chosen considering the lengths of our preferred stent (Neuroform Easy; Stryker), which were 15 and $20 \mathrm{~mm}$.

With this arbitrary segmentation, we placed six points along the centerline of the PA segment (Fig. 3B): P1 and $\mathrm{P} 2$ (points $7 \mathrm{~mm}$ and $3.5 \mathrm{~mm}$ proximal to the proximal margin of the ostium, respectively), P3 and P4 (proximal and distal points of the aneurysm ostium, respectively), and P5 and P6 (points $3.5 \mathrm{~mm}$ and $7 \mathrm{~mm}$ distal to the distal margin of the ostium, respectively). Using the analysis tool, we obtained the maximum and mean diameters and cross-sectional area of the PA at each point. The tortuosity index was calculated by 

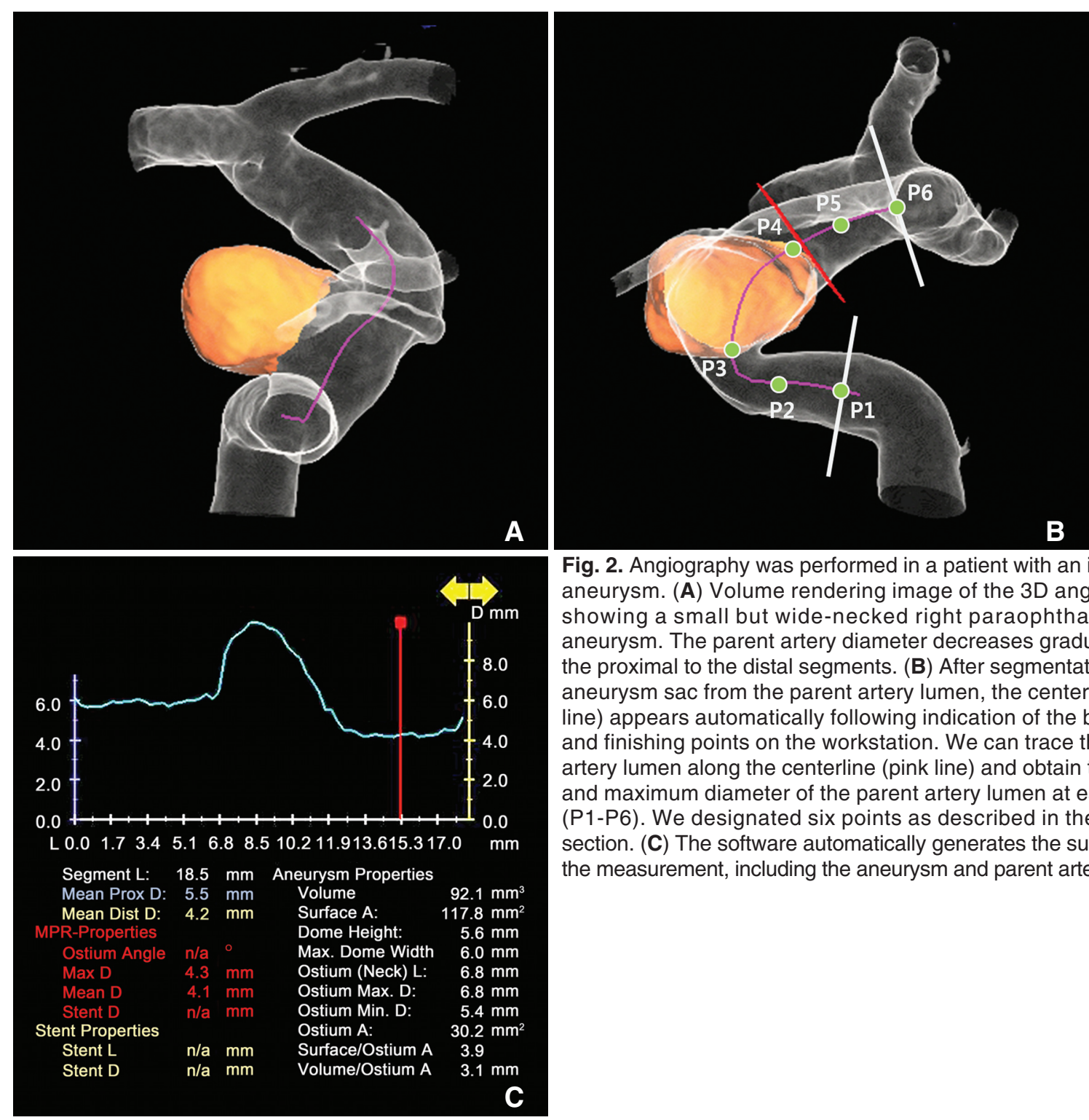

Fig. 2. Angiography was performed in a patient with an incidental aneurysm. (A) Volume rendering image of the 3D angiography showing a small but wide-necked right paraophthalmic ICA aneurysm. The parent artery diameter decreases gradually from the proximal to the distal segments. (B) After segmentation of the aneurysm sac from the parent artery lumen, the centerline (pink line) appears automatically following indication of the beginning and finishing points on the workstation. We can trace the parent artery lumen along the centerline (pink line) and obtain the mean and maximum diameter of the parent artery lumen at each point (P1-P6). We designated six points as described in the method section. (C) The software automatically generates the summary of the measurement, including the aneurysm and parent artery.
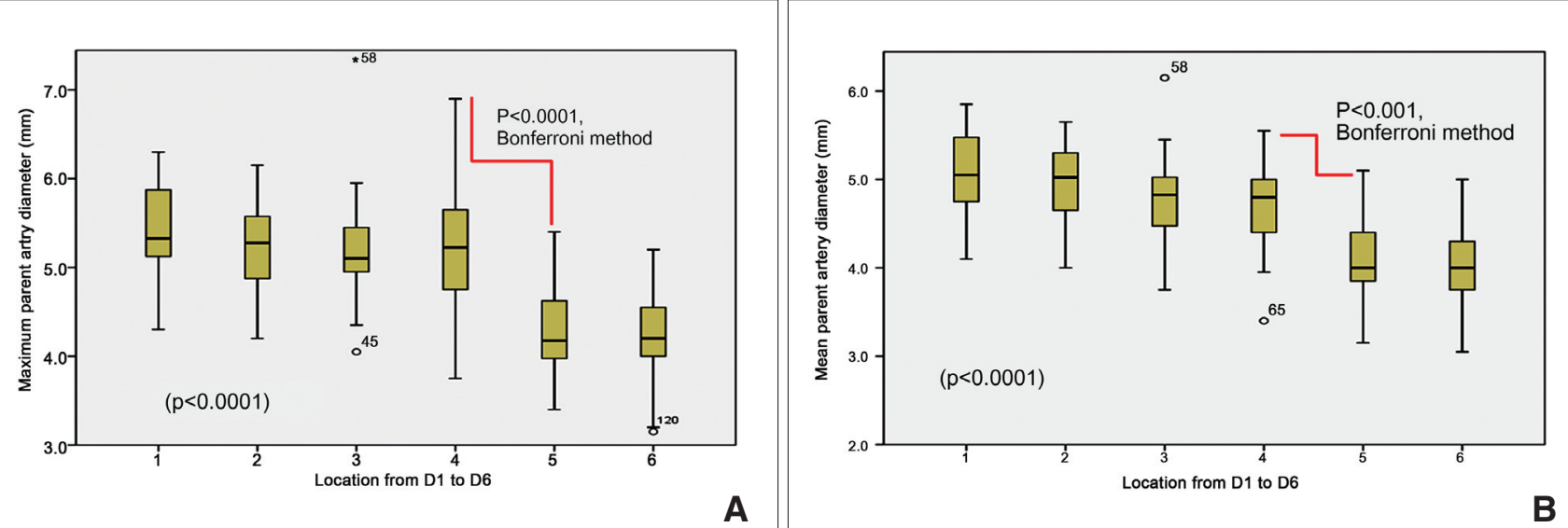

Fig. 3. Bar-and-whisker graphs of maximum $(\mathbf{A})$ and mean diameters of the parent artery $(\mathbf{B})$ from the most proximal point $(\mathrm{P} 1)$ to the most distal point (P6) of the parent artery segment. Significant diameter changes are noted between P4 and P5 in both graphs. 
dividing the distance from P1 to P6 along the centerline (centerline distance) by the direct distance (direct distance) from P1 to P6, respectively (Fig. 3B, 3C).

\section{Measurement and analysis}

After generation of the VR 3D image, each dataset was processed with a vessel analysis tool (Syngo AX Vessel Analysis, Siemens Medical Solutions). Analyses and measurements were performed by two radiology technicians, who had more than a year experience of using the software in their clinical practice. We measured the maximum and mean diameters at the six points of the PA as described above (Fig. 3).

We performed intra-class correlation analysis for the assessment of interobserver agreement between the two observers in measuring the mean diameters of the PA at each point. For statistical analysis, measurements by both observers were averaged. Statistical differences between the mean diameters of the six points were test by ANOVA. Post-hoc analyses were done using the Bonferroni method. Linear regression analyses were used to estimate independent contributions of morphologic variables of the PA dilatation. Variables with a probability value $\leq 0.1$ in univariate analyses were candidates for multivariate analyses. All statistical processing was performed with a statistical analysis package (SPSS ver. 11; Chicago, IL).

\section{RESULTS}

Of 26 consecutive cases, 20 distal ICA aneurysms were identified after exclusion. The patient population consisted of 15 women and 5 men with a median age of 56.5 years (range, 39-72). Morphologic parameters regarding the aneurysm sac, ostium, and its PA segment are summarized in Table 1. The intra-class correlation coefficient of the two observers in measuring the mean diameters of the PA at each point was $0.938(95 \%$ confidence interval: $0.910-0.958)$, which was excellent. The maximum PA diameters from P1 to P6 were $5.40 \mathrm{~mm}, 5.21 \mathrm{~mm}, 5.22 \mathrm{~mm}, 5.20 \mathrm{~mm}, 4.28 \mathrm{~mm}$, and $4.19 \mathrm{~mm}$, respectively. The mean PA diameters from P1 to P6 were $5.06 \mathrm{~mm}, 4.91 \mathrm{~mm}, 4.80 \mathrm{~mm}, 4.76$ $\mathrm{mm}, 4.08 \mathrm{~mm}$, and $4.01 \mathrm{~mm}$, respectively. The differences in diameter at each point between both measurements were statistically significant $(p<0.0001)$. In post-hoc analyses, the difference between P4 and P5 was significant both in maximum and mean PA diameters ( $p<0.0001$ and $p<0.001$, respectively; Fig. $3 \mathrm{~A}$ and $3 \mathrm{~B}$ ). Linear regression analysis did not reveal any significant morphological factors by multivariate analyses.

\section{DISCUSSION}

We observed a trend towards enlargement of the PA lumen at the aneurysmal segment in cases of widenecked ICA aneurysms, especially at the segment near the distal margin of the aneurysm ostium. This may be due to simple morphological influence secondary to the wide-neck of the aneurysm or to combined effect of morphologic change and chronic hemodynamic effects from the wide-necked aneurysm. Gradual widening of the aneurysm ostium may result in PA luminal deformities, as was previously reported [4]. This could be due to segmental vulnerability of the involved PA to mural changes that result in both arterial distension and aneurysm formation [5]. On the other hand, chronic

Table 1. Summary of the Aneurysms and Their Parent Artery Segments and Uni- and Multi-variate Analyses of Possible Influencing Morphologic Factors to Changes in Parent Artery Diameter

\begin{tabular}{|c|c|c|c|c|c|}
\hline & Parameter (units) & Median & Range & $\begin{array}{l}\text { Univariate analysis } \\
\quad(p \text { values })\end{array}$ & $\begin{array}{c}\text { Multivariate analysis } \\
\quad \text { ( } p \text { values) }\end{array}$ \\
\hline \multirow[t]{2}{*}{ Aneurysm sac } & Volume $\left(\mathrm{mm}^{3}\right)$ & 51.6 & $14.4-406.6$ & 0.486 & \\
\hline & Maximum diameter (mm) & 4.3 & $2.9-9.0$ & 0.318 & \\
\hline \multirow[t]{2}{*}{ Aneurysm ostium } & Maximum distance $(\mathrm{mm})$ & 5.2 & $3.8-8.2$ & 0.090 & 0.485 \\
\hline & Minimum distance (mm) & 4.2 & $2.8-7.4$ & 0.027 & 0.123 \\
\hline \multirow[t]{2}{*}{ Ratio } & Dome-to-neck ratio & 0.9 & $0.7-1.6$ & 0.646 & \\
\hline & Aspect ratio & 0.9 & $0.6-1.5$ & 0.870 & \\
\hline \multirow[t]{3}{*}{ Parent artery segment } & Centerline distance (mm) & 15.9 & $13.2-19.7$ & 0.739 & \\
\hline & Direct distance (mm) & 9.0 & $5.4-11.4$ & 0.732 & \\
\hline & Tortuosity index & 1.8 & $1.4-2.9$ & 0.537 & \\
\hline
\end{tabular}


mural abnormality of the vulnerable segment of the ICA may also be a basis for aneurysm formation [6].

Actually in the real clinical practice, due to the limitation of current stent-assisted coiling technique, we did not perform endovascular coiling in cases of a widenecked aneurysm which had significant dilatation of the PA mimicking a fusiform aneurysm. Those cases were not included in this analysis as they did not meet our arbitrary inclusion criteria. Inclusion of those cases would have prominently shown a trend of parent artery enlargement. The primary reason why those patients did not receive endovascular treatment was the current unavailability of a stent with a nominal diameter larger than $4.5 \mathrm{~mm}$ [7]. In such cases, the procedure can be performed using the largest possible self-expanding stent, since the unconstrained diameter is a little larger than the nominal diameter. However, there is risk of coil loop herniation between the potential space between the PA wall and stent mesh. Furthermore, since the stent is already fully expanded, very little hoof strength would remain to resist the herniating coil loops [7]. These cases would be good indications of flow diversion treatment.

In addition, poor wall apposition of the stent mesh is an important issue, not only as a mechanical issue but also from a hemodynamics and rheological perspective $[2,8]$. In this regard, we suggest the need of shapeadjusted stents to conform the parent artery morphology and securely protect the neck of the wide-necked aneurysms during coil insertion. The ideal shape of the stent could be fusiform or barrel-like with a middlesegment diameter that is larger than the edge segments.

We believe a similar issue should be considered for the use of flow diverters. Choosing an ideal size of flow diverter is difficult if the PA diameter varies significantly between the proximal segment, aneurysm segment, and distal segment. Poor device apposition to the wall can result in poor morphological and functional outcome [9]. Differing from the laser-cut, tube-slotted stent, the stent with a braided-mesh could adjust to the segmental difference in the parent artery diameter. However, change of the diameter at a certain segment of the parent artery could result in a change in cell-surface area or cell size of the device and eventually in unexpected alteration of local hemodynamics. Generation of a fusiform or barrel-shaped device could result in better performance in such a circumstances. The same analogy could be found in the proximal ICA where a tapered stent has successfully been applied to overcome the diameter difference between the distal CCA and proximal ICA [10].
There are several limitations to this study. First, our inclusion criteria may have been too limiting, which may have contributed to the small aneurysm sac size in our cohort. In addition, limiting inclusion to only cases of aneurysms where stent assistance was anticipated before the coiling procedure may have skewed the trend, as the indication is somewhat subjective according to the operator's preference. In addition to the limitation, because of the technical difficulty in the application of the vendor-provided software, we had no choice but to exclude several distal ICA aneurysm cases from the analyses. This might decrease the accuracy of our result and decrease representativeness of real clinical situation such as common association of distal ICA aneurysm with prominent branches, such as the posterior communicating artery, marked tortuosity of the parent artery, or an aneurysm with an irregularshaped sac, etc.

Furthermore, since we analyzed only the affected side of the ICA, we cannot eliminate the chance of differences between PA diameters at similar segments of the distal ICA without aneurysms. Further studies comparing PA diameters of the opposite segments of nonaffected distal ICA could help confirm our conclusion, which was drawn only with limited analysis of affected ICA only. Comparison of 3 groups (ICA with a widenecked aneurysm, ICA with a non-wide-necked aneurysm, and ICA out any aneurysm) in larger patient numbers would be a good methodological approach to support our results.

\section{CONCLUSION}

A parent artery harboring a wide-necked aneurysm requiring stent assistance for coiling shows a significantly larger parent artery diameter at the aneurysmal segment, especially at the distal transition segment of the aneurysm and the parent artery.

\section{References}

1. Lesley WS, Patel DV. Neuroform-EZ Stent Modification for Facilitated Delivery during Intracranial Aneurysm Embosurgery. Neurointervention 2013;8:101-104

2. Heller RS, Malek AM. Delivery technique plays an important role in determining vessel wall apposition of the enterprise selfexpanding intracranial stent. J Neurointerv Surg 2011;3:340-343

3. Turk AS, Strother CM, Crouthamel DI, Zagzebski JA. Definition of the ostium (neck) of an aneurysm revealed by intravascular sonography: an experimental study in canines. AJNR Am J Neuroradiol 1999;20:1301-1308

4. Yasuda R, Arat A, Strother CM, Aagaard-Kienitz B, Niemann D, 


\section{Jong Won Lee, et al.}

Mohamed A, et al. Aneurysm ostium angle: a predictor of the need for stent as assistance for endovascular aneurysm coiling in internal carotid artery sidewall aneurysms. AJNR Am J Neuroradiol 2011;32:1216-1220

5. Lasjaunias PL. Segmental identity and vulnerability in cerebral arteries. Interv Neuroradiol 2000;6:113-124

6. Farnoush A, Qian Y, Takao H, Murayama Y, Avolio A. Effect of saccular aneurysm and parent artery morphology on hemodynamics of cerebral bifurcation aneurysms. Conf Proc IEEE Eng Med Biol Soc 2012;2012:6677-6680

7. Krischek O, Miloslavski E, Fischer S, Shrivastava S, Henkes H. A comparison of functional and physical properties of self-expanding intracranial stents [Neuroform3, Wingspan, Solitaire, Leo+, Enterprise]. Minim Invasive Neurosurg 2011;54:21-28
8. Heller RS, Miele WR, Do-Dai DD, Malek AM. Crescent sign on magnetic resonance angiography revealing incomplete stent apposition: correlation with diffusion-weighted changes in stentmediated coil embolization of aneurysms. J Neurosurg 2011;115: 624-632

9. Ding D, Starke RM, Durst CR, Gaughen JR, Jr., Evans AJ, Jensen ME, et al. Dynact imaging for intraprocedural evaluation of flowdiverting stent apposition during endovascular treatment of intracranial aneurysms. J Clin Neurosci 2014;21:1981-1983

10. Matsumura JS, Gray W, Chaturvedi S, Yamanouchi D, Peng L, Verta P. Results of carotid artery stenting with distal embolic protection with improved systems: Protected Carotid Artery Stenting in Patients at High Risk for Carotid Endarterectomy (PROTECT) trial. J Vasc Surg 2012;55:968-976 